\title{
A stable ferryl porphyrin at the active site of Y463M BthA
}

Kimberly Rizzolo ${ }^{1}$, Andrew C. Weitz ${ }^{1,2}$, Steven E. Cohen ${ }^{3}$, Catherine L. Drennan ${ }^{3,4,5}$, Michael P. Hendrich $^{2}$, and Sean J. Elliott ${ }^{1 *}$

\author{
${ }^{1}$ Boston University, Dept. of Chemistry, Boston, MA 02215 \\ ${ }^{2}$ Carnegie Mellon University, Dept. of Chemistry, Pittsburgh, PA 15213 \\ ${ }^{3}$ Massachusetts Institute of Technology, Dept. of Chemistry, Cambridge, MA 02139 \\ ${ }^{4}$ Massachusetts Institute of Technology, Dept. of Biology, Cambridge, MA 02139 \\ ${ }^{5}$ Howard Hughes Medical Institute, Cambridge, MA 02139
}

\section{Table of Contents:}

\section{1) Methods}

- Expression and purification of Y463M

- UV-Visible Spectroscopy

- Steady-state kinetic assay

- Direct Electrochemistry

- Near-infrared spectroscopy

- Mössbauer and EPR spectroscopy of Y463M

- Crystallization of BthA Y463M

- Crystallographic Data Collection and Processing

- Model Building and Refinement

\section{2) Supplementary Figures}

- Figure S1: EPR data comparing Y463M treatments

- Figure S2: Optical absorption data comparing oxic and anoxic preparations, and CObound samples

- Figure S3: Mossbauer data and deconvolution of dithionite reduced samples

- Figure S4: Comparison of the 4.2K Mössbauer spectra of (A) dithionite-reduced Y463M BthA, and (B) anaerobically-purified Y463M.

- Figure S5: EPR of anaerobically purified Y463M

- Figure S6: 4.2K Mössbauer spectra and simulations of Y463M BthA

- Figure S7: An unmodelled region of electron density at the peroxidatic heme in chain B.

\section{3) Supplementary Tables}

- Table S1: Data collection and refinement statistics

- Table S2: Mössbauer fit parameters for species discussed in main text

\section{4) References}




\section{1) Methods}

Expression and purification of Y463M. Site-directed mutagenesis was carried out using Quik Change lightning II kit (Agilent), and the expression system for BthA from Burkholderia thailandensis described previously': the pETSN-based plasmid used here includes a leader sequence for expression in E. coli, and using S69 as the N-terminus of the protein, in order to avoid complications from $\mathrm{N}$-terminal processing in E. coli. Primer sequences used: ACCCTGCGTGACGCAGTGGCATTTATGAATACCCGCAGCACCGATCCGGCA (forward) and TGCCGGATCGGTGCTGCGGGTATTCATAAATGCCACTGCGTCACGCAGGGT (reverse). Mutation was verified by sequencing (GeneWiz). Expression and purification followed protocol previously described. ${ }^{1}$ Concentration of purified protein was determined by Bradford Assay. Samples were buffer exchanged into $50 \mathrm{mM}$ HEPES, $100 \mathrm{mM} \mathrm{NaCl}, 10 \%$ glycerol and kept at $-80^{\circ} \mathrm{C}$.

Anaerobic Purification of Y463M. Anaerobically prepared samples were grown identically to the aerobically prepared ${ }^{57} \mathrm{Fe}$-enriched Y463M BthA enzyme. Cells were harvested and flash frozen in liquid nitrogen. Frozen cell pellets were quickly transferred to a Coy anaerobic chamber (Coy Lab Products). Throughout the purification, the $\mathrm{O}_{2}$ concentration in the Coy Chamber was kept below $20 \mathrm{ppm}$. All consumables were introduced and equilibrated into the Coy chamber at least 4 days prior to the purification. $2 \mathrm{mg} / \mathrm{g}$ wet cell weight of DNAase and Lysozyme (Sigma Aldrich) were added to each cell pellet, along with $1 \mathrm{mM}$ PMSF. The pellets were resuspended in lysis buffer (50mM HEPES pH 7.8, $300 \mathrm{mM} \mathrm{NaCl}, 5 \% \mathrm{v} / \mathrm{v}$ glycerol) and lysed by sonication. Clarified lysate was obtained after centrifugation at $18,000 \times \mathrm{g}$ for $30 \mathrm{~min}$ in gas tight Nalgene centrifuge tubes. The clarified lysate was diluted by half in salt-free buffer $(50 \mathrm{mM}$ HEPES $\mathrm{pH}$ $7.8)$ to a final $[\mathrm{NaCl}]=150 \mathrm{mM}$, then loaded onto an SP Sepharose cation exchange resin column (GE Healthcare). After loading all protein, the column was washed with 3 column volumes buffer containing $150 \mathrm{mM} \mathrm{NaCl}$, then the protein was eluted with buffer containing $240 \mathrm{mM} \mathrm{NaCl}$. The fractions were collected and concentrated to approximately $1 \mathrm{~mL}$ sample volume, using Amicon Millipore $50 \mathrm{kDa}$ cutoff centrifuge filters. Purity of the eluted protein was confirmed by $12.5 \%$ SDS-PAGE gels. The purified protein was buffer exchanged to a final storage buffer $(50 \mathrm{mM}$ HEPES pH 7.8, $100 \mathrm{mM} \mathrm{NaCl}, 10 \% \mathrm{v} / \mathrm{v}$ glycerol). Electronic absorption spectra were taken for the anaerobically-purified Y463M BthA, confirming the protein was in the ferrous oxidation state. Air-exposure of the cuvette led to a shift in the Soret, consistent with that observed in the aerobically-purified samples.

Total protein concentration was quantified using the Bradford colormetric assay (BioRad). The total heme content for the diferric protein was measured for the WT enzyme using an extinction coefficient at $405 \mathrm{~nm}=208,000 \mathrm{M}^{-1} \mathrm{~cm}^{-1}$ (value reported as concentration of BthA monomers), and gave protein concentrations consistent with those measured by the Bradford assay for protein fractions with purity index $A_{405 n m} / A_{280 n m}>3$. The same extinction coefficient was found to correlate well with the protein concentration measured by the Bradford assay for the aerobically prepared Y463M variant, after treatment with sodium ascorbate.

UV-Visible Spectroscopy. UV-visible spectra for the oxidized and reduced state of BthA and variants were obtained on a Cary 50 spectrophotometer (Agilent) using $1 \mathrm{~mL}$ quartz cuvettes (Starna cells). The oxidized state was determined under aerobic conditions at $21.0^{\circ} \mathrm{C}$ in $50 \mathrm{mM}$ HEPES, $50 \mathrm{mM} \mathrm{NaCl}$, pH 7.8 buffer. For reduction of Y463M $(5 \mu \mathrm{M})$ with sodium ascorbate, reductant was added to the cuvettes to a final concentration of $250 \mu \mathrm{M}$ under anaerobic conditions with the use of gas-tight syringes (Hamilton). Reduction with ascorbate was achieved after incubation of protein with 50 equivalents ascorbate for $1 \mathrm{hr}$ at $21^{\circ} \mathrm{C}$. Data were background 
subtracted by taking the average absorbance from $780-800 \mathrm{~nm}$ and subtracting this value from each data point.

Steady-state kinetic assay. Peroxidase activity was determined using 2,2'-azino-bis(3ethylbenzothiazoline-6-sulphonic acid) (ABTS, Sigma) as the artificial electron donor. Assay was performed aerobically in assay buffer $(50 \mathrm{mM}$ phosphate, $\mathrm{pH} 6.5)$ at $21.0^{\circ} \mathrm{C}$, using hydrogen peroxide concentrations ranging from $0.3 \mu \mathrm{M}$ to $30 \mu \mathrm{M}$. ABTS and $\mathrm{H}_{2} \mathrm{O}_{2}$ were reacted for 10 seconds before addition of enzyme, and background reactivity was subtracted from each trace. Enzyme was added to final concentration of $60 \mathrm{nM}$ (determined by Bradford). The increase of absorbance at $420 \mathrm{~nm}$ was monitored with extinction coefficient $\varepsilon_{420}=36 \mathrm{mM}^{-1} \mathrm{~cm}^{-1}$. Data were recorded on a Cary50 spectrophotometer (Agilent).

Direct Electrochemistry. Electrochemistry experiments were performed using a three component electrode system in an anaerobic chamber (MBraun). Set-up consisted of a saturated calomel electrode (Fisher) and platinum wire counter electrode. Pyrolytic graphite edge (PGE) electrodes were used as the working electrode. To prepare the PGE electrode, the graphite surface was sanded and polished with an aqueous mix of $1 \mu \mathrm{M}$ alumina before sonication in Milli-Q water for 2 minutes. Polished electrodes were then let to dry before depositing $16 \mu \mathrm{L}$ of multiwalled carbon nanotube (MWCNT) mixture. Mixture consisted of $1 \mathrm{mg}$ MWCNT (sigma) in 1 $\mathrm{mL}$ of DMF. Solution was sonicated for 15 minutes before pipetted onto the polished PGE surface. Electrodes were left to dry overnight to ensure surface was sufficiently coated and DMF was no longer present. Before depositing protein, electrodes were rinsed excessively with water and left to dry. For preparation of adsorbed protein onto the MWCNT-coated surface, $1-4 \mu \mathrm{L}$ of $\sim 400 \mu \mathrm{M}$ protein was pipetted onto the electrode and left to stand for 30 seconds before excess protein was pipetted off. Cell solution consisted of multicomponent buffer [ $5 \mathrm{mM}$ HEPES, MES, MOPS, CAPS, TAPS and CHES, $100 \mathrm{mM} \mathrm{NaCl}$ ] to allow for collection at varying $\mathrm{pH}$ from 5.0 to 9.5. Experiments were carried out at $21^{\circ} \mathrm{C}$. Measurements were recorded on a PGSTAT 12 Autolab (Ecochemie) potentiostat. Data were analyzed using QSoas (Fourmond 2016).

Near-infrared spectroscopy. Near-IR spectra were recorded on a Shimadzu 3000 spectrophotometer. Protein $(5 \mu \mathrm{M})$ was prepared in $50 \mathrm{mM}$ HEPES, pH 7.8. Spectra were collected at $21^{\circ} \mathrm{C}$ in $1 \mathrm{~mL}$ quartz cuvettes (Starna Cells). For $\mathrm{H}_{2} \mathrm{O}_{2}$ treated samples, $\mathrm{H}_{2} \mathrm{O}_{2}$ was added to a final concentration of $50 \mu \mathrm{M} . \mathrm{H}_{2} \mathrm{O}_{2}$ stock solutions were prepared in MQ water from $30 \%(\mathrm{w} / \mathrm{v})$ reagent grade stock (Sigma Aldrich). Cuvette was immediately inverted after addition of $\mathrm{H}_{2} \mathrm{O}_{2}$ and scanned immediately.

Mössbauer and EPR spectroscopy of Y463M. Incorporation of ${ }^{57} \mathrm{Fe}$ was achieved through expression of Y463M in minimal media (MM) as described for Wt BthA. ${ }^{157} \mathrm{Fe}$ metal (Cambridge Isotopes) was added to a final concentration of $2 \mathrm{mg} / \mathrm{L}$. Expression of ${ }^{57} \mathrm{Fe}-\mathrm{Y} 463 \mathrm{M}$ was carried out following protocol for Wt BthA. ${ }^{57} \mathrm{Fe}-\mathrm{Y} 463 \mathrm{M}$ final protein sample was prepared in a final sample buffer [50 mM HEPES, $100 \mathrm{mM} \mathrm{NaCl}, 10 \%$ glycerol, $\mathrm{pH} 7.8$ ].

X-band EPR spectra were recorded on a Bruker Elexsys spectrometer equipped with an Oxford ESR 900 cryostat and a Bruker ER 4122 SHQ cavity for generation of microwave fields transverse to the applied magnetic field. The modulation amplitude and frequency were $1 \mathrm{mT}$ and $100 \mathrm{kHz}$ for all spectra. The microwave frequency was calibrated with a frequency counter and the magnetic field was calibrated with a calibrated Hall probe. The temperature was calibrated with a carbon glass resistor (Lakeshore CGR-1-1000). EPR signals were quantified relative to a 1 $\mathrm{mM} \mathrm{Cu}$ (II)EDTA standard in $10 \%$ glycerol, prepared from a commercially bought $\mathrm{Cu}$ (II) AAS standard solution (Sigma Aldrich). Species concentrations were determined with the software SpinCount developed by M. P. Hendrich. ${ }^{2}$ The software diagonalizes the spin Hamiltonian, 


$$
H=\beta_{\mathrm{e}} \mathbf{S} \cdot \mathrm{g}_{\mathrm{e}} \cdot \mathbf{B}+\mathbf{S} \cdot \mathbf{D} \cdot \mathbf{S},
$$

where all parameters have their usual definitions. The quantitative simulations are least-squares fits of the experimental spectra generated with consideration of all intensity factors, which allows the computation of simulated spectra for a specified sample concentration.

${ }^{57} \mathrm{Fe}$ Mössbauer spectra were recorded using a Janis Research dewar. Isomer shifts are reported relative to Fe metal at $298 \mathrm{~K}$. The simulations of Mössbauer spectra were calculated with leastsquares fitting using the program SpinCount and the standard spin Hamiltonian ${ }^{2}$ :

$$
\begin{aligned}
H= & \beta_{\mathrm{e}} \mathbf{S} \cdot \mathrm{g}_{\mathrm{e}} \cdot \mathbf{B}+\mathrm{D}\left[\boldsymbol{S}_{\mathrm{z}}{ }^{2}-\mathrm{S}(\mathrm{S}+1) / 3+\mathrm{E} / \mathrm{D}\left(\mathbf{S}_{\mathbf{x}}{ }^{2}-\boldsymbol{S}_{\mathrm{y}}{ }^{2}\right)+\mathbf{S} \cdot \mathrm{A} \cdot \mathbf{I}\right. \\
& -\mathrm{g}_{\mathrm{n}} \beta_{\mathrm{n}} \mathbf{B} \cdot \mathbf{I}+\mathrm{eQ} \mathrm{V}_{\mathrm{zz}} / 12\left[3 \mathbf{I}_{\mathbf{z}}{ }^{2}-\mathrm{I}(\mathrm{I}+1)+\eta\left[\mathbf{I}_{\mathbf{x}}{ }^{2}-\mathbf{I}_{\mathbf{y}}{ }^{2}\right]\right]
\end{aligned}
$$

Crystallization of Y463M. Initial crystals of Y463M were identified using the Index HT (Hampton Research) and MCSG1 (Anatrace) suites, and all optimization and cryoprotectant solutions were prepared using chemicals purchased from Hampton Research. Crystal conditions for $\mathrm{Y} 463 \mathrm{M}$ were identified through sparse matrix screening at $21^{\circ} \mathrm{C}$ with an $\mathrm{Nt} 8$ (Formulatrix) using a microseed stock generated from crystals of wild-type BthA as described previously. ${ }^{1}$ Initial crystals formed from $150 \mathrm{~nL}$ of Y463M $(9 \mathrm{mg} / \mathrm{mL}$ in $50 \mathrm{mM}$ HEPES, $\mathrm{pH} 7.8 ; 100 \mathrm{mM}$ $\mathrm{NaCl} ; 5 \%$ (v/v) glycerol) that was added to $250 \mathrm{~nL}$ of precipitant solution (100 mM Bis-Tris, $\mathrm{pH}$ $6.5 ; 200 \mathrm{mM}\left(\mathrm{NH}_{4}\right)_{2} \mathrm{SO}_{4}, 25 \%$ PEG 3350) and $50 \mathrm{~nL}$ wild-type of BthA microseed stock and equilibrated against a reservoir of $70 \mu \mathrm{L}$ precipitant solution using the sitting drop method. After 2 weeks, red crystals grew out of a light precipitate to form stacks of plates. Through successive rounds of rescreening, microseeding, and grid screening, final crystals were grown by adding 1 $\mu \mathrm{L}$ Y 463M $(10 \mathrm{mg} / \mathrm{mL}$ in $50 \mathrm{mM}$ HEPES, $\mathrm{pH} 7.8,100 \mathrm{mM}$ sodium chloride, $5 \%$ (v/v) glycerol) to $0.8 \mu \mathrm{L}$ precipitant solution (100 mM Tris, $\mathrm{pH} 8.5 ; 160 \mathrm{mM} \mathrm{MgCl} 2 ; 18 \%$ (w/v) PEG $4000 ; 20 \%$ $(\mathrm{v} / \mathrm{v})$ glycerol) and $0.2 \mu \mathrm{L}$ Y $463 \mathrm{M}$ microseed stock dissolved in the same precipitant solution and equilibrating against $500 \mu \mathrm{L}$ precipitant solution using the sitting drop method. Red rod-like crystals grew between 1 and 3 weeks from a light precipitate. Crystals were harvested and flash frozen in liquid nitrogen.

Crystallographic Data Collection and Processing. A native data set of Y463M was collected at a wavelength of $0.9791 \AA$ and temperature of $100 \mathrm{~K}$ on a Pilatus $6 \mathrm{M}$ pixel detector on beamline 24ID-C at the Advanced Photon Source (Argonne, IL). The data were indexed, integrated, and scaled in spacegroup $\mathrm{P} 2{ }_{1} 2_{1} 2_{1}$ with cell edges $\mathrm{a}=49.97 \AA, \mathrm{b}=87.61 \AA$, and $\mathrm{c}=184.17 \AA$ to 1.59 $\AA$ resolution using HKL2000. ${ }^{3}$

Model Building and Refinement. The structure of Y463M was determined to 1.59- $\AA$ resolution by molecular replacement in PHASER ${ }^{4}$. The structure of wild-type BthA (PDB: 6NX0) was used as a search model. ${ }^{1}$ All non proteinogenic atoms were removed from the model and Y463 was stubbed to the $\alpha$-carbon. A solution with 2 molecules per asymmetric unit was found with LLG and TFZ scores of 12,527 and 98.3 , respectively. The initial model had values of $37.7 \%$ and $37.3 \%$ for the working and free $R$-factors, respectively. Iterative rounds of model building and refinement were performed in $\mathrm{COOT}^{5}$ and phenix.refine ${ }^{6}$, respectively. Two heme $c$ cofactors were added and covalently linked to C188 and C191 as well as C367 and C370 of each protomer using a parameter file. Heme planarity restraints were relaxed to allow for heme ruffling. ${ }^{7}$ Once the polypeptide chain and heme cofactors were built, waters were added automatically and curated manually. Riding hydrogen atoms were added to the protein model using phenix.ready_set ${ }^{6}$. Later refinements and the final structure were verified using composite omit maps. 
Chain A of the final model contains residues 125-543 of 543 and two covalently bonded two covalently bonded $c$-type heme cofactors. Chain B of the final model contains residues 125-543 of 543 and two covalently bonded two covalently bonded $c$-type heme cofactors. The periplasmic localization sequence (vide infra) was not part of the crystallization construct and the first 59 residues of both protomers are not modeled due to a lack of electron density. Analysis of the final model was performed with MolProbity ${ }^{8}$, identifying $97.48 \%, 2.40 \%$, and $0.12 \%$ of residues in the favored, allowed, and disallowed regions of the Ramachandran plot. The only Ramachandran outlier is a glycine, G144/B. Rotamer analysis shows $0.77 \%$ rotamer outliers. M368/A, F480/A, T157/B, M368/B, and F480/B are listed as rotamer outliers, but the electron density supports how these side chains were modeled.

Although an $\mathrm{Fe}(\mathrm{IV})=\mathrm{O}$ species is reported at the peroxidatic heme in as-isolated $\mathrm{Y} 463 \mathrm{M}$ BthA protein, such a species is not observed crystallographically, as it may degrade during the nearly month-long process of protein crystallization, or due to reduction in the X-ray beam. The region near the open coordination site of the peroxidatic heme and P271 on chain A contains two ordered water molecules (Figure S7C) and the analogous region on chain B contains unmodelled electron density (Figure S7D). This region is consistent with either a di- or triatomic molecule, but is too far from the heme iron to be consistent with peroxide, carbon monoxide or nitric oxide binding. Additionally, the crystallization condition contains no molecules consistent with the observed density. This density could be due to the combination of multiple ordered water networks, the $\mathrm{Fe}(\mathrm{IV})=\mathrm{O}$ species, and beam-reduced species all at partial occupancies."

The data collection, processing, and refinement statistics are summarized in Supplementary Table S1. Figures were generated using PyMOL (Schrödinger, LLC). All structural software packages were compiled by SBGrid. ${ }^{9}$ The atomic coordinates and structure factors for Y463M have been deposited in the Protein Data Bank (https://www.wwpdb.org/) under the accession code 6V59. 


\section{2) Supplementary Figures}

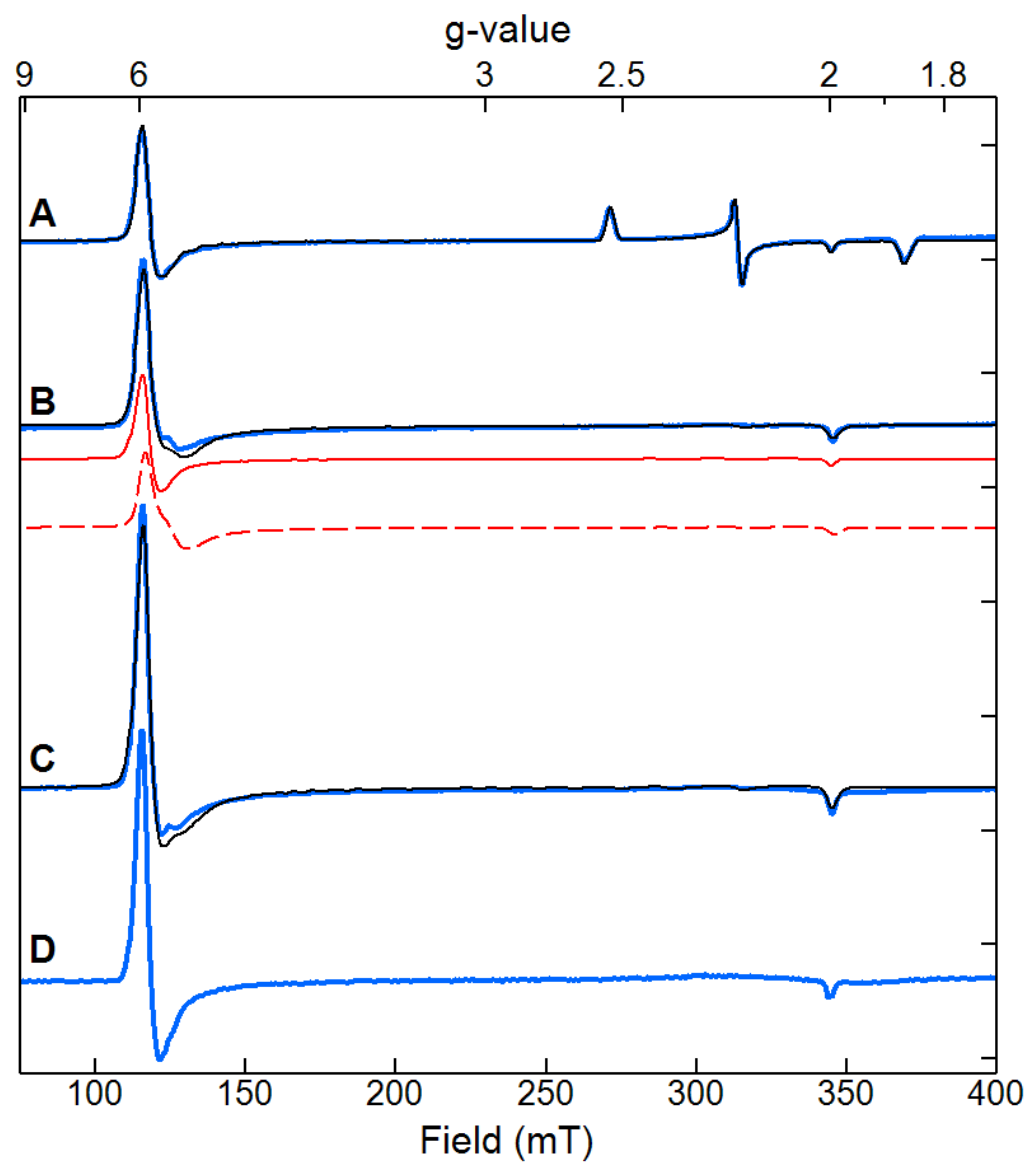

Figure S1: X-band EPR spectra (blue) a recorded at $12 \mathrm{~K}$ of as-isolated and ascorbate-treated WT and Y463M samples, and fits (black). (A) As-isolated WT BthA, consisting of near-equal ratios of HS $(\mathrm{g}=6)$ and LS $(\mathrm{g}=2.55,2.19,1.86) \mathrm{Fe}(\mathrm{III})$ hemes as previously reported. (B) Asisolated, aerobically-purified Y463M BthA $(1.1 \mathrm{mM})$. This sample was prepared in parallel with the Mössbauer sample from Figure 3B in the main text. The LS species from WT is not present in this variant, and instead shows a more rhombic HS Fe(III) species. The sum of least-squares simulations (black trace) for the axial peroxidatic heme (solid red trace, $0.22 \mathrm{mM}$ ), and the rhombic HS Fe(III) species (dashed red trace, $0.36 \mathrm{mM}$ ) are fit to the data and are consistent with the corresponding amounts in the Mössbauer sample shown in Figure 3B. (C) Y463M BthA (0.7 $\mathrm{mM}$ ), from B, with 20eq sodium ascorbate added. No LS Fe(III) has appeared in the signal; instead the axial HS Fe(III) species $(0.32 \mathrm{mM})$ grows in intensity relative to the rhombic species $(0.23 \mathrm{mM}$ ). These assignments leave approximately $0.15 \mathrm{mM}$ (or $\sim 20 \%$ ) of protein unaccounted for. Similarly, there is about $\sim 12 \%$ of iron unaccounted for in the fits for the parallel Mössbauer sample in Figure 3C. This could be attributed to some integer spin species, but the noise of the Mössbauer data prohibits a precise assignment. Nevertheless, the two spectra clearly show ascorbate treatment gives rise to near-equal amounts of the axial HS Fe(III) in both EPR and Mössbauer samples. (D) Difference spectrum of (C-B), corrected for sample concentration, highlighting the growth of an axial HS Fe(III) species similar to the 5c peroxidatic site of WT BthA following ascorbate addition to Y463M BthA, consistent with the observations in Figure $3 \mathrm{C}$. 

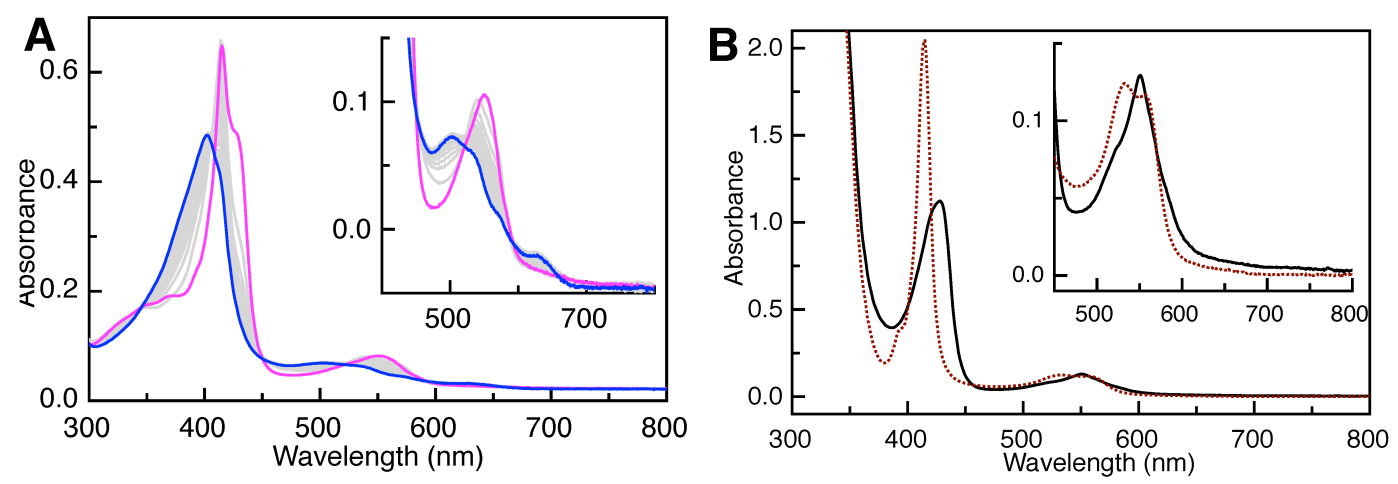

Figure S2: (A) Electronic Absorption spectra of anaerobically purified Y463M BthA (pink trace) and the air-exposed sample (blue trace). The gray traces represent intermediate spectra collected every $1.5 \mathrm{~min}$ after air exposure. The anaerobic sample shows strong features at 416 and $427 \mathrm{~nm}$, which decay to give rise to a Soret band near $403 \mathrm{~nm}$ following air exposure. (B) Electronic absorption spectra of dithionite-reduced WT BthA (solid line) and after bubbling of CO (g) for $30 \mathrm{~s}$ (red dots). The Soret shifts from $428 \mathrm{~nm}$ in the diferrous sample to $416 \mathrm{~nm}$ in the CO-bound sample. Comparison of these spectra to (A) suggest the anaerobically-purified Y463M BthA contains a mixture of reduced heme and CO-bound heme, consistent with the Mössbauer observations in Figure 4. 


\section{Deconvolution of the Mössbauer spectra from Figure 4.}

To better deconvolute the Mössbauer spectrum of the anaerobically-purified Y463M BthA sample, dithionite reduced samples of WT and Y463M BthA (Figure S3) were prepared and Mössbauer spectra recorded at $4.2 \mathrm{~K}$ with an applied field of $45 \mathrm{mT} \| \gamma$. The Mössbauer spectrum of dithionite-reduced WT BthA was fit with two doublets (A, black trace): (1) $\delta=0.57 \mathrm{~mm} / \mathrm{s}$, $\Delta \mathrm{E}_{\mathrm{Q}}=1.64 \mathrm{~mm} / \mathrm{s}$ (solid red trace, $41 \%$ of total Fe); and (2) $\delta=0.89 \mathrm{~mm} / \mathrm{s}, \Delta \mathrm{E}_{\mathrm{Q}}=2.33 \mathrm{~mm} / \mathrm{s}$ (dashed red trace, $57 \%$ ). The outer doublet (2) had parameters with typical high spin (HS) $\mathrm{S}=2$ ferrohemes, and thus was assigned to the catalytic 5c His-coordinated heme. The inner doublet then was assigned to the 6c His/Tyr heme, yet has an isomer shift higher than most known low spin (LS) $\mathrm{S}=0$ ferrohemes $(0.41 \mathrm{~mm} / \mathrm{s}<\delta<0.51 \mathrm{~mm} / \mathrm{s})$, but also two low for HS ferrous hemes. In addition, the quadrupole splitting is much higher than in other LS ferrohemes $(0.75 \mathrm{~mm} / \mathrm{s}<$ $\left.\Delta \mathrm{E}_{\mathrm{Q}}<1.44 \mathrm{~mm} / \mathrm{s}\right){ }^{10}$ Relatively little is known about the Mössbauer properties of His/Tyr coordinated hemes, but the data suggest this heme is not LS in the ferrous state. These results are more consistent with intermediate spin $(\mathrm{S}=1)$ ferrohemes, though these are relatively uncommon outside of synthetic model complexes. Since most LS ferrous heme complexes are six coordinate, the larger quadrupole splitting parameters of the Tyr/His heme in BthA relative to other ferrohemes suggests possibly one of the coordinating amino acids is weakly coordinated to the $\mathrm{Fe}(\mathrm{II})$. In support of this, the crystal structure data for the diferric WT enzyme indicated a long Fe- $\mathrm{O}_{\text {Tyr }}$ bond length, that while stabilizes a LS configuration in the Fe(III) oxidation state, could favor a intermediate or HS configuration in the ferrous oxidation state. Mössbauer spectra of this sample at higher magnetic fields could allow correct assignment of the spin state for the His/Tyr heme, but this was beyond the scope of this paper.

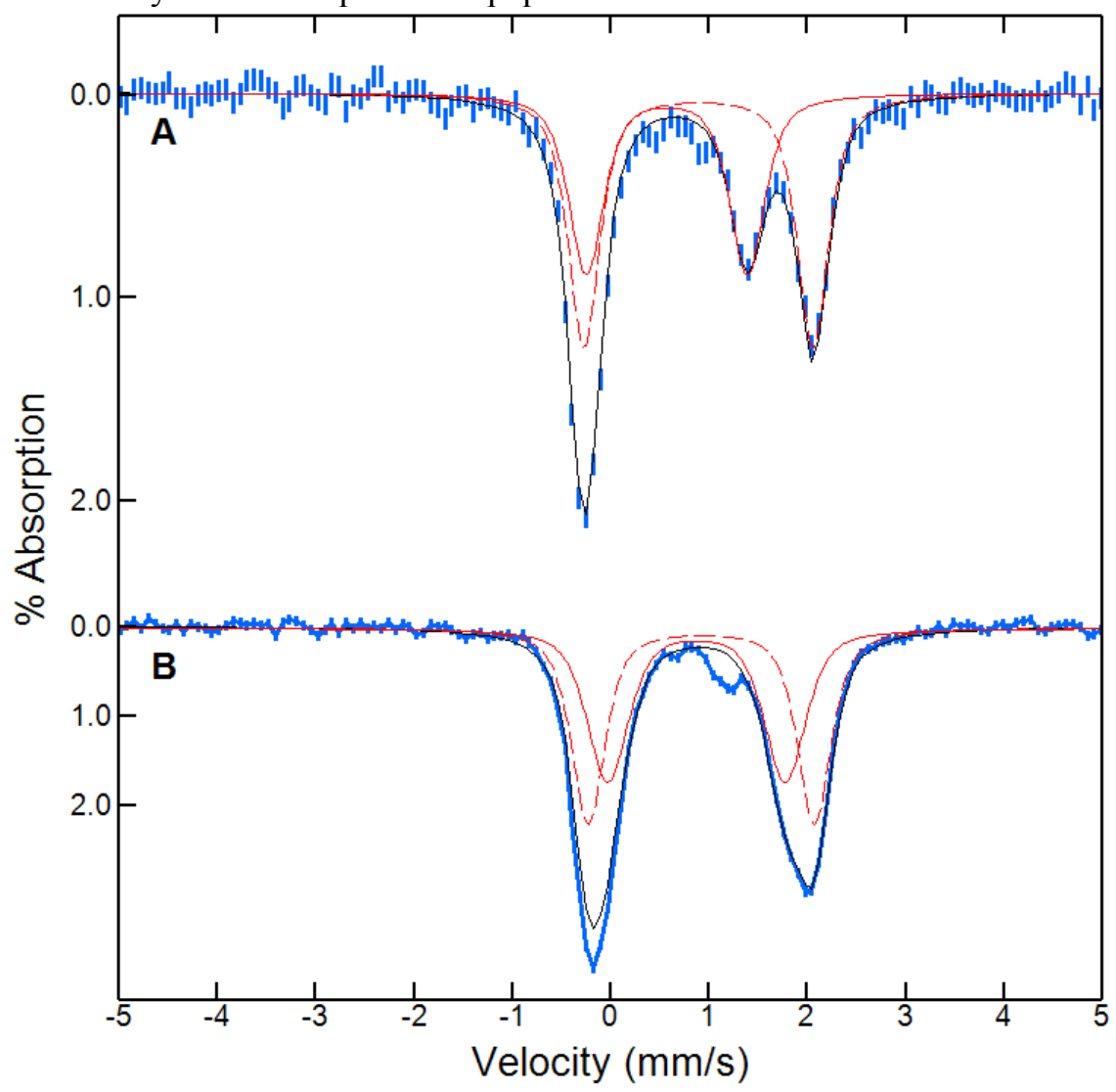

Figure S3. 4.2 K, $45 \mathrm{mT}(\mathrm{B} \| \gamma)$ Mössbauer spectra of dithionite-reduced WT (A) and Y463M (B) BthA samples. The blue vertical bars represent the data, the black trace is the sum of the inner (dashed red trace) and outer (solid red trace) doublets, representing the $6 \mathrm{c}$ and $5 \mathrm{c}$ hemes, respectively. 
The Mössbauer and (to a better extent) the EPR spectra of Y463M BthA demonstrate that the electronic properties of the peroxidatic (5c) heme remain largely unchanged relative to WT; that is, highly axial HS Fe(III). Spectra of Y463M following ascorbate addition revealed a HS Fe(III) signal that nearly overlaps with that found in WT (Figures 3 and S1). The similarity of the spectral features for the $5 \mathrm{c}$ heme in the Fe(III) oxidation state suggested the quadrupole splitting parameters may also be similar for this heme in the Fe(II) state. Consequently, constraints were placed while fitting the Mössbauer spectra of dithionite-reduced Y463M in Figure S3 to restrict the quadrupole splitting parameters of the ferrous catalytic heme to match closely to those from the corresponding heme in WT. The dithionite-reduced Y463M BthA spectrum showed one broad doublet that was deconvoluted using the aforementioned constraint (Figure S3B): (1) $\delta=$ $0.87 \mathrm{~mm} / \mathrm{s}, \Delta \mathrm{E}_{\mathrm{Q}}=1.80 \mathrm{~mm} / \mathrm{s}$ (solid red trace, $45 \%$ of total Fe); and (2) $\delta=0.92 \mathrm{~mm} / \mathrm{s}, \Delta \mathrm{E}_{\mathrm{Q}}=2.30$ $\mathrm{mm} / \mathrm{s}$ (dashed red trace, $47 \%$ ). The outer doublet's (2) parameters closely match those for $5 \mathrm{c}$ Hiscoordinated catalytic heme; therefore the inner doublet (1) is assigned to the His/Met-coordinated heme. The large isomer shift and quadrupole splitting of this doublet has parameters to suggest a high spin configuration, in contrast to known His/Met coordinated hemes such as the diheme becp from Paracoccus denitrificans $\left(\delta=0.42 \mathrm{~mm} / \mathrm{s}, \Delta \mathrm{E}_{\mathrm{Q}}=1.27 \mathrm{~mm} / \mathrm{s}\right)$ that are low spin. ${ }^{11}$ The quadrupole splitting parameters and proposed HS configuration of the His/Met heme of Y463M are consistent with this heme as HS in the ferric oxidation state. The HS configuration in both oxidation states are supported by the crystal structure showing a long Fe-Met bond. There is a remaining $8 \%$ of area that could be accounted for by fitting a doublet with $\delta=0.49 \mathrm{~mm} / \mathrm{s}$ and $\Delta \mathrm{E}_{\mathrm{Q}}=1.79 \mathrm{~mm} / \mathrm{s}$, however its origin was unclear, and was not included in the fit of the data.

The doublets obtained from dithionite-reduced Y463M were then used to fit the spectrum of the anaerobically-purified Y463M sample (Figure S4). The anaerobically-prepared sample shows a loss in the outer doublet relative to the inner, in addition to growth of a new doublet with $\delta=0.27$ $\mathrm{mm} / \mathrm{s}$ and $\Delta \mathrm{E}_{\mathrm{Q}}=0.41 \mathrm{~mm} / \mathrm{s}$, as well as a minority paramagnetic species. The new doublet has parameters highly similar to CO-bound heme adducts. ${ }^{10}$ The paramagnetic species were identified in a parallel EPR sample (Figure S5) as a mixture of HS Fe(III) $(0.005 \mathrm{mM}, 2.5 \%$ of protein in sample) and a low spin nitric oxide (NO)-bound heme adduct $\left(\mathrm{g}_{\|}=2.068, \mathrm{~g}_{\perp}=2.0,0.043 \mathrm{mM}, 22\right.$ $\%$ of protein in sample). The spin quantification of these two species in the corresponding EPR sample is in agreement with the area unaccounted for in fits of the Mössbauer sample (25\%). A subsequent sample of dithionite-reduced Y463M + excess $\mathrm{NO}(\mathrm{g}$ ) yielded a $\mathrm{S}=1 / 2$ species identical to that found in the anaerobic sample, confirming the identity of the $S=1 / 2$ feature as an $\left\{\mathrm{FeNO}^{7}\right.$ adduct (Figure S5). We cannot explain the origin of NO (possibly during cell lysis, from which we attributed the CO-bound species), but the high similarity in EPR signatures between the anaerobically prepared and NO-treated Y463M samples supports an NO-bound heme species. 


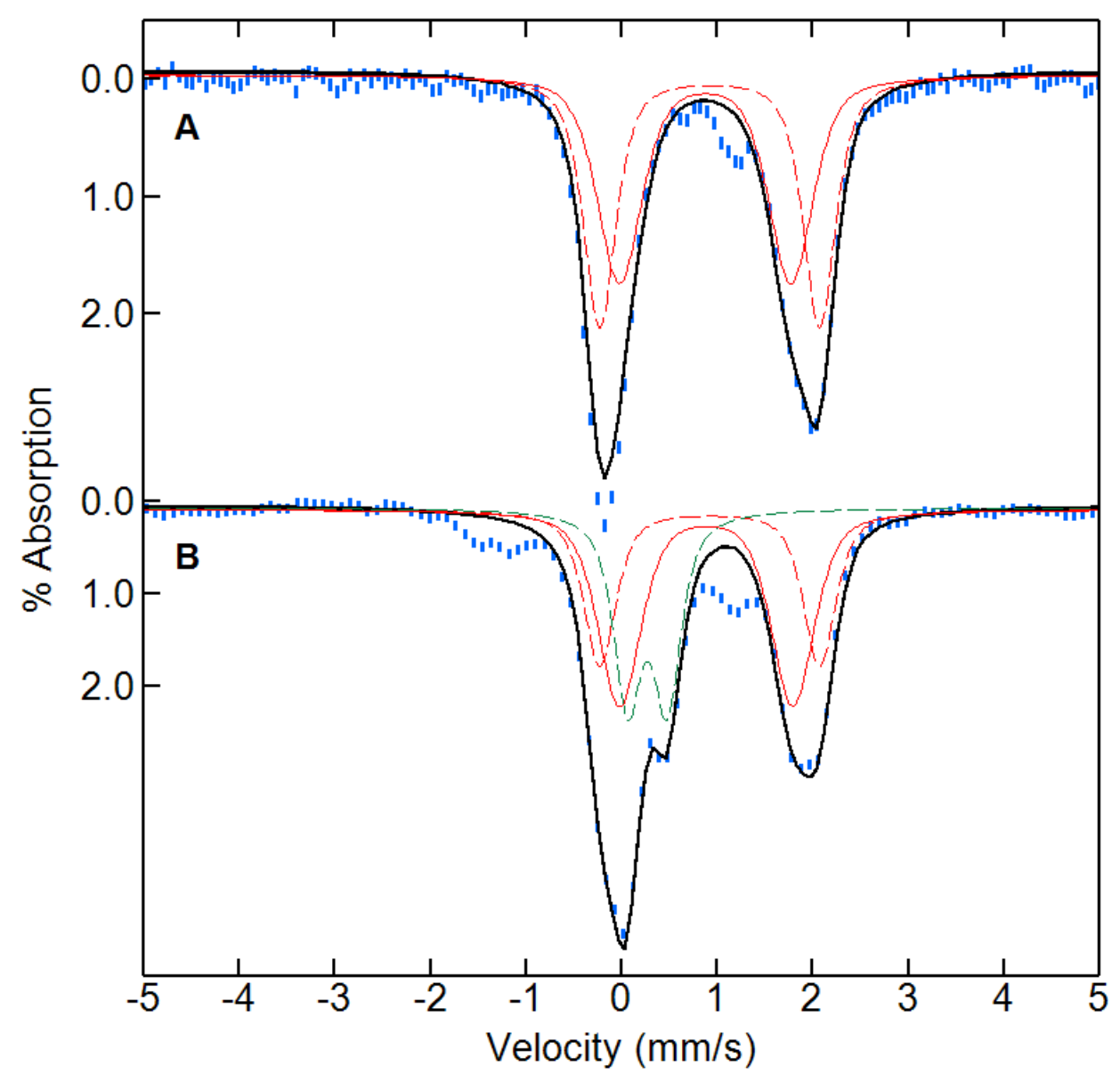

Figure S4: Comparison of the 4.2K Mössbauer spectra of (A) dithionite-reduced Y463M BthA, and (B) anaerobically-purified Y463M. In comparison to (A), spectrum (B) shows relatively less of the $5 \mathrm{c} \mathrm{Fe}(\mathrm{II})$ heme (dashed red trace), and appearance of narrow doublet with parameters consistent with $\mathrm{Fe}(\mathrm{II}) \mathrm{CO}$ heme (dashed green trace). Unaccounted for area in (B) is due to trace paramagnetic species, accounting for approximately $25 \%$ of the total $\mathrm{Fe}$. 


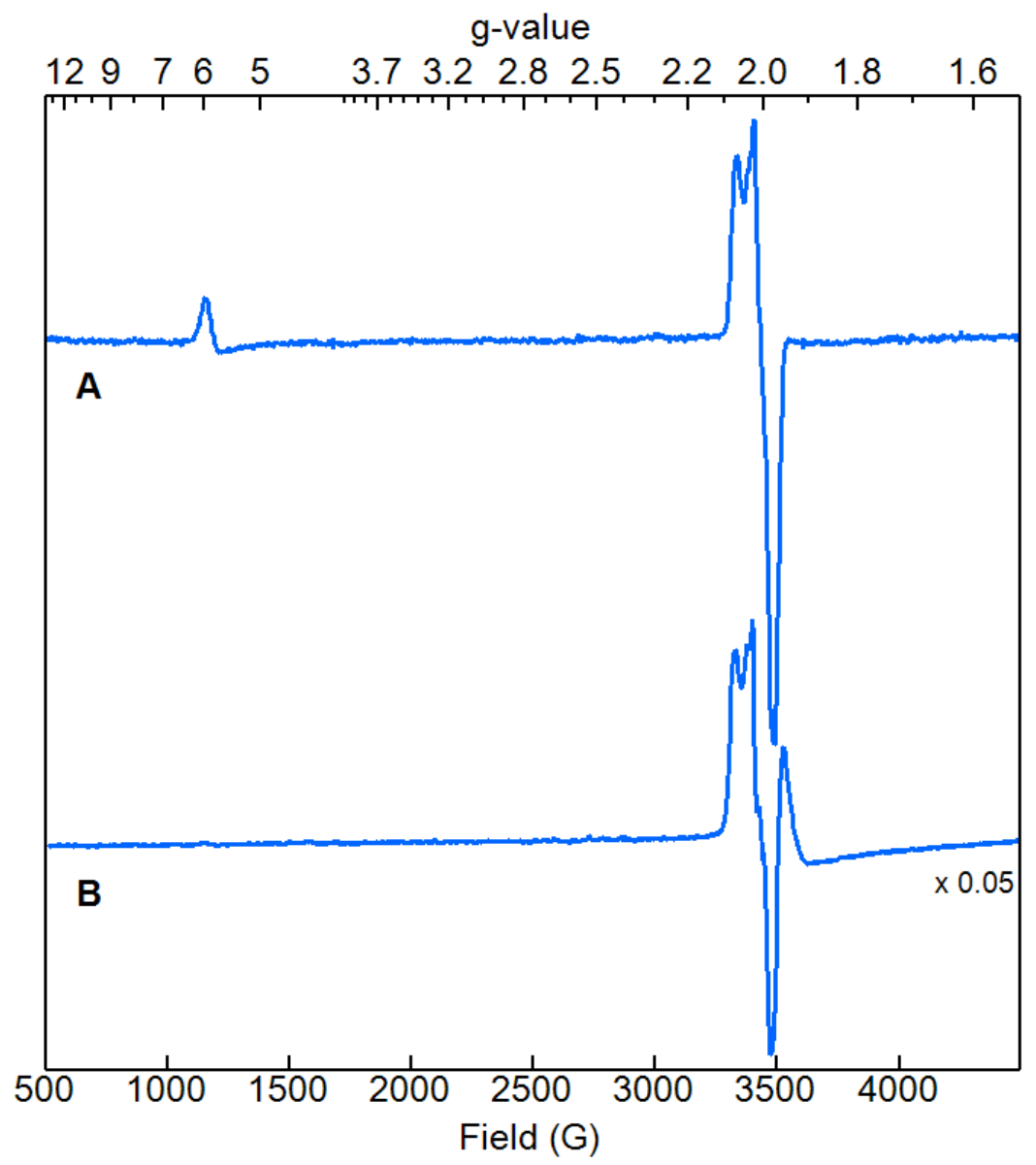

Figure S5: $15 \mathrm{~K}$ X-band EPR data of the anaerobically-purified Y463M BthA (A), and dithionite-reduced Y463M BthA + 2eq proliNONOate (B, scaled by $1 / 20)$. The anaerobicallypurified Y463M BthA spectrum shows two features, near $g=6$, and near $g=2$. The $g=6$ feature is from a HS Fe(III) signal. The $\mathrm{g}=2$ signal was identified as a $\mathrm{S}=1 / 2\{\mathrm{FeNO}\}^{7}$ complex, and reproduced by addition of 2eq proliNONOate to dithionite-reduced Y463M BthA. Double integrations of the signals are consistent with $25 \%$ yield of paramagnetic species in the Mössbauer sample corresponding to spectrum (A) and 100\% of the expected protein concentration in (B). The additional feature below $\mathrm{g}=2$ in (B) is from excess NO generated in the solution. 


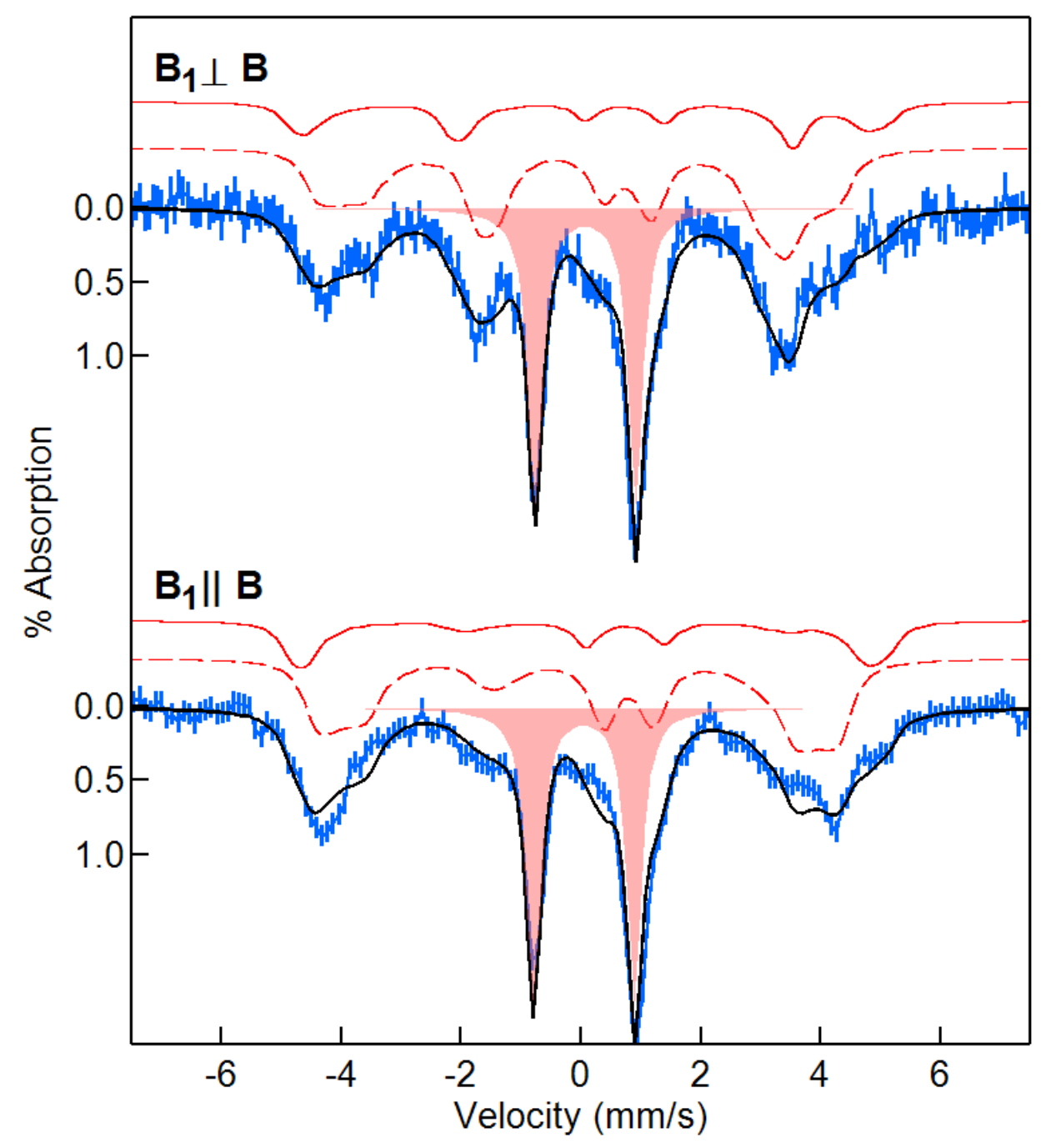

Figure S6. 4.2 K, $45 \mathrm{mT}$ Mössbauer spectra of aerobically purified Y463M BthA from Figure 3B in main text. (A) $\mathrm{B} \perp \gamma$. (B) $\mathrm{B} \| \gamma$. The blue vertical bars represent the data, the solid black trace is the sum of the peroxidatic heme (solid red trace) and Met/His heme (dashed red trace) of Y364M $\mathrm{BthA}$, and the $\mathrm{Fe}(\mathrm{IV}) \mathrm{O}$ species (filled red doublet). The parameters for theY463M Met/His heme are listed in Table S2 (here we used $\mathrm{D}=10 \mathrm{~cm}^{-1}, \mathrm{E} / \mathrm{D}=0.018, \mathrm{~A}_{\mathrm{x}, \mathrm{y}}=-15(1),-17(1)$ ), , and the fit for the peroxidatic heme is from the simulations for WT BthA (Weitz, A.C.; Biswas, S.; Rizzolo, K.; Elliott, S. J.; Bominaar, E.L.; Hendrich, M.P. The Electronic state of the His/Tyr-ligated heme of BthA by Mössbauer and DFT analysis, in preparation ). The fits are not meant to be exhaustive (the simulations of the Met/His heme are not sensitive to changes in $A_{z}$ under these conditions), but still can adequately capture the change in speciation discussed in Figure 3. 


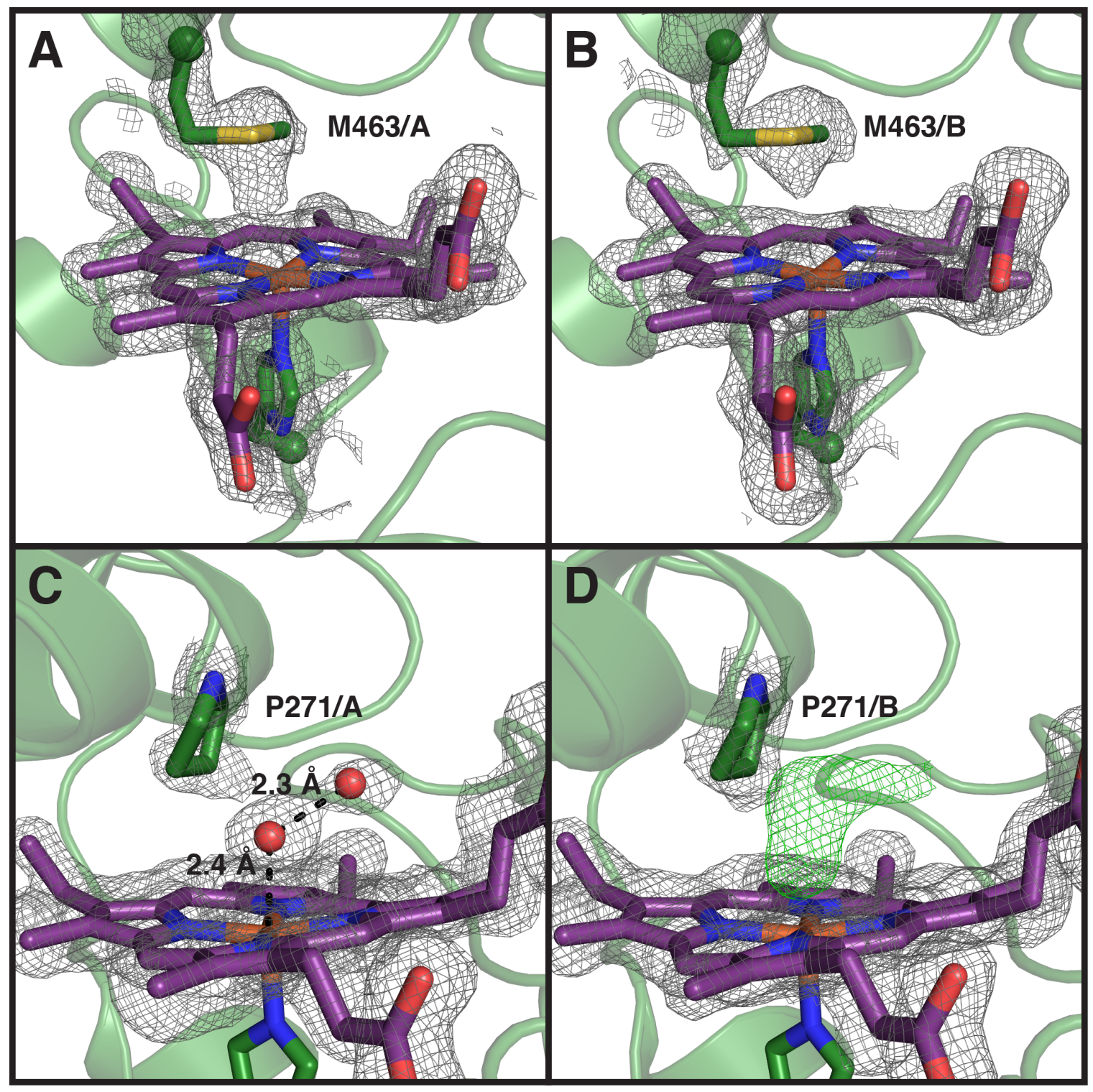

Figure S7: The heme pockets within the two molecules of Y463M BthA that are present in the asymmetric unit. (A) Electron density around the $\delta$-sulfur of Met463 is stretched, but sulfur is not bound to the heme Fe in chain A. (B) Electron density indicates that Met463 is not bound to the heme Fe in chain B either. This panel is rotated slightly from view of chain B that shown in Figure 2B. The terminal methyl group of the Met side chain is visible in this orientation. (C) Electron density is not consistent with an $\mathrm{Fe}(\mathrm{IV})=\mathrm{O}$ species at the peroxidatic heme in chain $\mathrm{A}$. A distance of $2.4 \AA$ between the $\mathrm{Fe}$ and the presumed oxygen atom is too long for an $\mathrm{Fe}(\mathrm{IV})=\mathrm{O}$ species, but this distance is consistent with an Fe coordinated water molecule. The electron density suggests that this water molecule is part of an ordered water network. (D) Un-modeled electron density at the peroxidatic heme in chain $\mathrm{B}$ is not consistent with an $\mathrm{Fe}(\mathrm{IV})=\mathrm{O}$ species. This density is consistent with either a di- or triatomic molecule, and is too far from the heme iron to be peroxide, carbon monoxide or nitric oxide. Structures depicted as in Figure 2 with $2 F_{O}-F_{C}$ composite omit electron density contoured at $1 \sigma$ and $F_{O}-F_{C}$ positive difference electron density shown in green and contoured at $3 \sigma$. 
3) Supplementary Tables

Table S1: Data collection and refinement statistics

\begin{tabular}{ll}
\hline & Y $463 \mathrm{M}$ \\
\hline Data collection & \\
Space group & P2 $2{ }_{1}$ \\
Cell dimensions & \\
$\quad a, b, c(\AA)$ & $49.97,87.61,184.2$ \\
Resolution $(\AA)$ & $50.00-1.59$ \\
$R_{\text {sym }}$ & $9.5(48.2)^{*}$ \\
No. reflections & 708,344 \\
Unique reflections & 107,369 \\
Redundancy & $6.6(5.6)$ \\
Completeness $(\%)$ & $98.8(94.0)$ \\
$C C 1 / 2$ & $(0.884)$ \\
$<I>/<\sigma I>$ & $16.3(2.8)$ \\
Refinement & \\
Resolution $(\AA)$ & $48.23-1.59$ \\
$R_{\text {work }} / R_{\text {free }}$ & $15.10 / 17.74$ \\
No. atoms & \\
Protein & 12,504 \\
Heme & 300 \\
Glycerol & 147 \\
Magnesium & 1 \\
Chloride & 4 \\
Water & 920 \\
$B$-factors $\left(\AA{ }^{2}\right)$ & \\
Protein & 23.2 \\
Heme & 16.1 \\
Glycerol & 36.4 \\
Magnesium & 34.8 \\
Chloride & 30.8 \\
Water & 31.2 \\
R.m.s. deviations & \\
$\quad$ Bond lengths $(\AA)$ & 0.005 \\
$*$ Values in parentheses are for highest-resolution shell. \\
\end{tabular}


Table S2. Experimental parameters for species found in WT and Y463M BthA

\begin{tabular}{|c|c|c|c|c|c|c|c|}
\hline & Spin & $\begin{array}{c}\delta \\
\mathrm{mm} / \mathrm{s}\end{array}$ & $\begin{array}{c}\Delta \mathrm{E}_{\mathrm{Q}} \\
\mathrm{mm} / \mathrm{s}\end{array}$ & $\begin{array}{l}\% \text {-age, } \\
\text { Fig 3B }\end{array}$ & $\begin{array}{l}\% \text {-age, } \\
\text { Fig 3C }\end{array}$ & $\begin{array}{l}\% \text {-age, } \\
\text { Fig } 4 \mathrm{~A}\end{array}$ & $\begin{array}{l}\% \text {-age, } \\
\text { Fig } 4 \mathrm{~B}\end{array}$ \\
\hline $\begin{array}{l}\text { WT BthA, } \\
\text { peroxidatic }^{1,2}\end{array}$ & $5 / 2$ & 0.42 & +1.37 & 27 & 47 & & \\
\hline $\begin{array}{l}\text { WT BthA, } \\
\text { His/Tyr }{ }^{1}\end{array}$ & $1 / 2$ & 0.21 & -2.67 & - & - & & \\
\hline $\begin{array}{l}\text { Y463M, } \\
\text { Met/Tyr }\end{array}$ & $5 / 2$ & 0.38 & 1.9 & 41 & 41 & & 31 \\
\hline $\mathrm{Fe}(\mathrm{IV}) \mathrm{O}$ & n.d. & 0.08 & 1.67 & 33 & - & & 20 \\
\hline $\begin{array}{l}\text { Reduced WT, } \\
\text { peroxidatic }\end{array}$ & n.d. & 0.89 & 2.33 & & & & \\
\hline $\begin{array}{l}\text { Reduced WT, } \\
\text { His/Tyr }\end{array}$ & n.d. & 0.57 & 1.64 & & & & \\
\hline $\begin{array}{l}\text { Reduced Y463M, } \\
\text { peroxidatic }\end{array}$ & n.d. & 0.92 & $2.30^{3}$ & & & 42 & \\
\hline $\begin{array}{l}\text { Reduced Y463M, } \\
\text { His/Met }\end{array}$ & n.d. & 0.87 & $1.80^{3}$ & & & 27 & \\
\hline $\mathrm{Fe}(\mathrm{II}) \mathrm{CO}$ & n.d. & 0.25 & $0.49(5)$ & & & 23 & 28 \\
\hline oxyheme & n.d. & 0.27 & 2.41 & & & & 21 \\
\hline
\end{tabular}

${ }^{1}$ Rigorous calculation of these values are presented in an upcoming manuscript in preparation. (Weitz, A.C.; Biswas, S.; Rizzolo, K.; Elliott, S. J.; Bominaar, E.L.; Hendrich, M.P.;

The Electronic state of the His/Tyr-ligated heme of BthA by Mössbauer and DFT analysis., in preparation $)^{2}$ The fit for the peroxidatic heme in WT is used to fit the peroxidatic heme in Y463M, shown in Figure 3. ${ }^{3}$ Values reported in Figure S3, they are shifted somewhat from the least-squares fits of Figure 4, but are in general agreement. 


\section{4) References}

1. Rizzolo, K.; Cohen, S. E.; Weitz, A. C.; López Muñoz, M. L.; Hendrich, M. P.; Drennan, C. L.; Elliott, S. J., A widely distributed diheme enzyme from Burkholderia that displays an atypically stable bis-Fe(IV) state. Nature Communications 2019, 10, 1101.

2. Petasis, D. T.; Hendrich, M. P., Quantitative Interpretation of Multifrequency Multimode EPR Spectra of Metal Containing Proteins, Enzymes, and Biomimetic Complexes. Methods Enzymol 2015, 563, 171-208.

3. Otwinowski, Z.; Minor, W., Processing of X-ray diffraction data collected in oscillation mode. Methods Enzymol 1997, 276, 307-26.

4. $\quad$ McCoy, A. J.; Grosse-Kunstleve, R. W.; Adams, P. D.; Winn, M. D.; Storoni, L. C.; Read, R. J., Phaser crystallographic software. J Appl Crystallogr 2007, 40 (Pt 4), 658674.

5. Emsley, P.; Cowtan, K., Coot: model-building tools for molecular graphics. Acta Crystallogr D Biol Crystallogr 2004, 60 (Pt 12 Pt 1), 2126-32.

6. Adams, P. D.; Afonine, P. V.; Bunkoczi, G.; Chen, V. B.; Davis, I. W.; Echols, N.; Headd, J. J.; Hung, L. W.; Kapral, G. J.; Grosse-Kunstleve, R. W.; McCoy, A. J.; Moriarty, N. W.; Oeffner, R.; Read, R. J.; Richardson, D. C.; Richardson, J. S.; Terwilliger, T. C.; Zwart, P. H., PHENIX: a comprehensive Python-based system for macromolecular structure solution. Acta Crystallogr D Biol Crystallogr 2010, 66 (Pt 2), 213-21.

7. $\quad$ Roberts, S. A.; Weichsel, A.; Qiu, Y.; Shelnutt, J. A.; Walker, F. A.; Montfort, W. R., Ligand-induced heme ruffling and bent no geometry in ultra-high-resolution structures of nitrophorin 4. Biochemistry 2001, 40 (38), 11327-37.

8. Chen, V. B.; Arendall, W. B., 3rd; Headd, J. J.; Keedy, D. A.; Immormino, R. M.; Kapral, G. J.; Murray, L. W.; Richardson, J. S.; Richardson, D. C., MolProbity: all-atom structure validation for macromolecular crystallography. Acta Crystallogr D Biol Crystallogr 2010, 66 (Pt 1), 12-21.

9. Morin, A.; Eisenbraun, B.; Key, J.; Sanschagrin, P. C.; Timony, M. A.; Ottaviano, M.; Sliz, P., Collaboration gets the most out of software. Elife 2013, 2, e01456.

10. Debrunner, P. G., Mössbauer spectroscopy of iron porphyrins. Physical Bioinorganic Chemistry Series 1989, 4 (Iron Porphyrins, Pt. 3), 137-234.

11. Prazeres, S.; Moura, J. J. G.; Moura, I.; Gilmour, R.; Goodhew, C. F.; Pettigrew, G. W.; Ravi, N.; Huynh, B. H., Mössbauer characterization of Paracoccus denitrificans cytochrome c peroxidase. Further evidence for redox and calcium binding-induced heme-heme interaction. J. Biol. Chem. 1995, 270 (41), 24264-9. 